\title{
Helical Morphologies of Thermotropic Liquid-Crystalline Chiral Schiff-based Rod-coil Amphiphiles
}

\author{
Tz-Feng Lin ${ }^{\dagger}$, Rong-Ming Ho ${ }^{\dagger}$, Chien-Hung Sung ${ }^{\ddagger}$ Chain-Shu Hsu *t $^{*}$ \\ ${ }^{\dagger}$ Department of Chemical Engineering, National Tsing-Hua University, Hsinchu 30013, Taiwan, \\ R.O.C. \\ ${ }^{\ddagger}$ Department of Applied Chemistry, National Chiao Tung University, Hsinchu 30050, Taiwan, R.O.C. \\ e-mail: rmho@mx.nthu.edu.tw; cshsu@mail.nctu.edu.tw
}

\section{Supporting Information}

Table S1. The enthalpy of the phase transitions of chiral Schiff-based rod-coil amphiphiles studied.

Figure S1 Thermograms of LC7 were performed in different heating rate.

Figure S2 Thermogram of crystallization studies were performed in different temperature through $55^{\circ} \mathrm{C}$ to $135{ }^{\circ} \mathrm{C}$ by LC11. A crystallization window was found between $65{ }^{\circ} \mathrm{C}$ and $130{ }^{\circ} \mathrm{C}$, and the maximum crystallization rate temperature was identified at $95{ }^{\circ} \mathrm{C}$.

Figure S3 Well-defined smectic-like structure observed by SAED [00l] zone diffraction.

Figure S4 FESEM image of fractured banded texture at room temperature. 
Table S1. The enthalpy of the phase transitions of chiral Schiff-based rod-coil amphiphiles studied.

\begin{tabular}{|c|c|c|c|}
\hline \multirow{2}{*}{\multicolumn{2}{|c|}{$\begin{array}{c}\text { Entry } \\
\text { (scanning rate, }{ }^{\circ} \mathrm{C} / \mathrm{min} \text { ) }\end{array}$}} & \multicolumn{2}{|c|}{ Enthalpy (J/g) } \\
\hline & & cooling & heating \\
\hline \multirow{5}{*}{ LC7 } & 2.5 & $\mathrm{I}-1.10, \mathrm{SmA}-80.68, \mathrm{~K}$ & K 87.11, SmA 4.49, I \\
\hline & 5 & I -1.16, SmA -81.98, K & K 87.93, SmA 4.66, I \\
\hline & $10^{\mathrm{c}}$ & I - 2.25, SmA -93.62, K & K 110.49, SmA 5.40, I \\
\hline & 20 & I -2.96 SmA -93.21, K & K 98.64, SmA 5.62, I \\
\hline & 40 & I -3.14, SmA - $94.55, \mathrm{~K}$ & K 102.36, SmA 5.81, I \\
\hline \multirow{5}{*}{ LC9 } & 2.5 & $\mathrm{I}-1.42, \mathrm{SmA}-, \mathrm{SmC}^{*}-5.50, \mathrm{~K}-, \mathrm{T}_{\mathrm{g}}$ & $\mathrm{T}_{\mathrm{g}}$ 一, $\mathrm{K}-16.50, \mathrm{SmC}^{*} 23.90, \mathrm{SmA} 2.44, \mathrm{I}$ \\
\hline & 5 & $\mathrm{I}-1.48, \mathrm{SmA}-, \mathrm{SmC}^{*}-5.53, \mathrm{~K}-, \mathrm{T}_{\mathrm{g}}$ & $\mathrm{T}_{\mathrm{g}}-, \mathrm{K}-22.37, \mathrm{SmC}^{*} 28.16, \mathrm{SmA} 2.69, \mathrm{I}$ \\
\hline & $10^{\mathrm{c}}$ & $\mathrm{I}-2.97, \mathrm{SmA}-, \mathrm{SmC}^{*}-19.73, \mathrm{~K}-, \mathrm{T}_{\mathrm{g}}$ & $\mathrm{T}_{\mathrm{g}}$ 一, K -20.59, SmC* 24.30, SmA 2.93, I \\
\hline & 20 & I -2.98 SmA 一, $\mathrm{SmC}^{*}-16.90, \mathrm{~K}-, \mathrm{T}_{\mathrm{g}}$ & $\mathrm{T}_{\mathrm{g}}-, \mathrm{K}-22.25, \mathrm{SmC}^{*} 31.97, \mathrm{SmA} 3.40, \mathrm{I}$ \\
\hline & 40 & $\mathrm{I}-3.06, \mathrm{SmA} \longrightarrow, \mathrm{SmC}^{*}-18.74, \mathrm{~K} \longrightarrow, \mathrm{T}_{\mathrm{g}}$ & $\mathrm{T}_{\mathrm{g}}$-, K -21.10, SmC* 29.34, SmA 3.54, I \\
\hline \multirow{5}{*}{$\mathrm{LC} 11^{\mathrm{b}}$} & 2.5 & $\mathrm{I}-1.90, \mathrm{SmA} \longrightarrow, \mathrm{SmC}^{*}-9.38, \mathrm{~K} \longrightarrow, \mathrm{T}_{\mathrm{g}}$ & $\mathrm{T}_{\mathrm{g}}$ 一, $\mathrm{K}-28.38, \mathrm{SmC}^{*} 26.13, \mathrm{SmA} 2.03, \mathrm{I}$ \\
\hline & 5 & $\mathrm{I}-1.89, \mathrm{SmA}-, \mathrm{SmC}^{*}-8.98, \mathrm{~K}-, \mathrm{T}_{\mathrm{g}}$ & $\mathrm{T}_{\mathrm{g}}-, \mathrm{K}-22.34, \mathrm{SmC}^{*} 27.50, \mathrm{SmA} 2.01, \mathrm{I}$ \\
\hline & $10^{\mathrm{c}}$ & $\mathrm{I}-2.44, \mathrm{SmA}-, \mathrm{SmC}^{*}-13.40, \mathrm{~K}-, \mathrm{T}_{\mathrm{g}}$ & $\mathrm{T}_{\mathrm{g}}-, \mathrm{K}-24.82, \mathrm{SmC}^{*} 27.82, \mathrm{SmA} 2.56, \mathrm{I}$ \\
\hline & 20 & $\mathrm{I}-2.48, \mathrm{SmA}-, \mathrm{SmC}^{*}-14.43, \mathrm{~K}-, \mathrm{T}_{\mathrm{g}}$ & $\mathrm{T}_{\mathrm{g}}$ 一, K -29.61, SmC* 27.99, SmA 2.17, I \\
\hline & 40 & $\mathrm{I}-2.53, \mathrm{SmA}-, \mathrm{SmC}^{*}-20.50, \mathrm{~K}-, \mathrm{T}_{\mathrm{g}}$ & $\mathrm{T}_{\mathrm{g}}$ 一, K -34.34, SmC* 28.65, SmA 2.79, I \\
\hline \multirow{5}{*}{ LC13 } & 2.5 & $\mathrm{I}-1.25, \mathrm{SmA}-, \mathrm{SmC}^{*}-16.88, \mathrm{~K}-, \mathrm{T}_{\mathrm{g}}$ & $\mathrm{T}_{\mathrm{g}}$ 一, $\mathrm{K}-10.12, \mathrm{SmC}^{*} 30.61, \mathrm{SmA} 1.39, \mathrm{I}$ \\
\hline & 5 & $\mathrm{I}-1.29, \mathrm{SmA}-, \mathrm{SmC}^{*}-26.10, \mathrm{~K}-, \mathrm{T}_{\mathrm{g}}$ & $\mathrm{T}_{\mathrm{g}}-, \mathrm{K}-19.41, \mathrm{SmC}^{*} 46.65, \mathrm{SmA} 1.49, \mathrm{I}$ \\
\hline & $10^{\mathrm{c}}$ & $\mathrm{I}-1.85, \mathrm{SmA} \longrightarrow, \mathrm{SmC}^{*}-27.89, \mathrm{~K} \longrightarrow, \mathrm{T}_{\mathrm{g}}$ & $\mathrm{T}_{\mathrm{g}}$ 一, K -32.36, SmC*66.71, SmA 1.96, I \\
\hline & 20 & $\mathrm{I}-1.74 \mathrm{SmA}-, \mathrm{SmC}^{*}-36.98, \mathrm{~K}-, \mathrm{T}_{\mathrm{g}}$ & $\mathrm{T}_{\mathrm{g}}$ 一, $\mathrm{K}-36.66, \mathrm{SmC}^{*}$ 78.14, SmA 1.32, I \\
\hline & 40 & $\mathrm{I}-1.76, \mathrm{SmA}-, \mathrm{SmC}^{*}-38.46, \mathrm{~K}-, \mathrm{T}_{\mathrm{g}}$ & $\mathrm{T}_{\mathrm{g}}$-, $\mathrm{K}-40.41, \mathrm{SmC}^{*} 74.33, \mathrm{SmA} 1.60, \mathrm{I}$ \\
\hline
\end{tabular}

${ }^{\mathrm{a}} \mathrm{I}=$ isotropic phase; $\mathrm{SmA}=$ smectic A phase; $\mathrm{SmC}^{*}=$ chiral smectic $\mathrm{C}$ phase; $\mathrm{K}=$ crystalline phase; $\mathrm{T}_{\mathrm{g}}=$ glass transition. Transition temperatures $\left({ }^{\circ} \mathrm{C}\right)$ are determined by the on-set temperature of DSC profiles.

${ }^{\mathrm{b}}$ Thermograms of DSC cooling and heating scans are demonstrated in Figure 1.

${ }^{\mathrm{c}}$ Thermograms of DSC cooling and heating scans are demonstrated in Figure 2. 


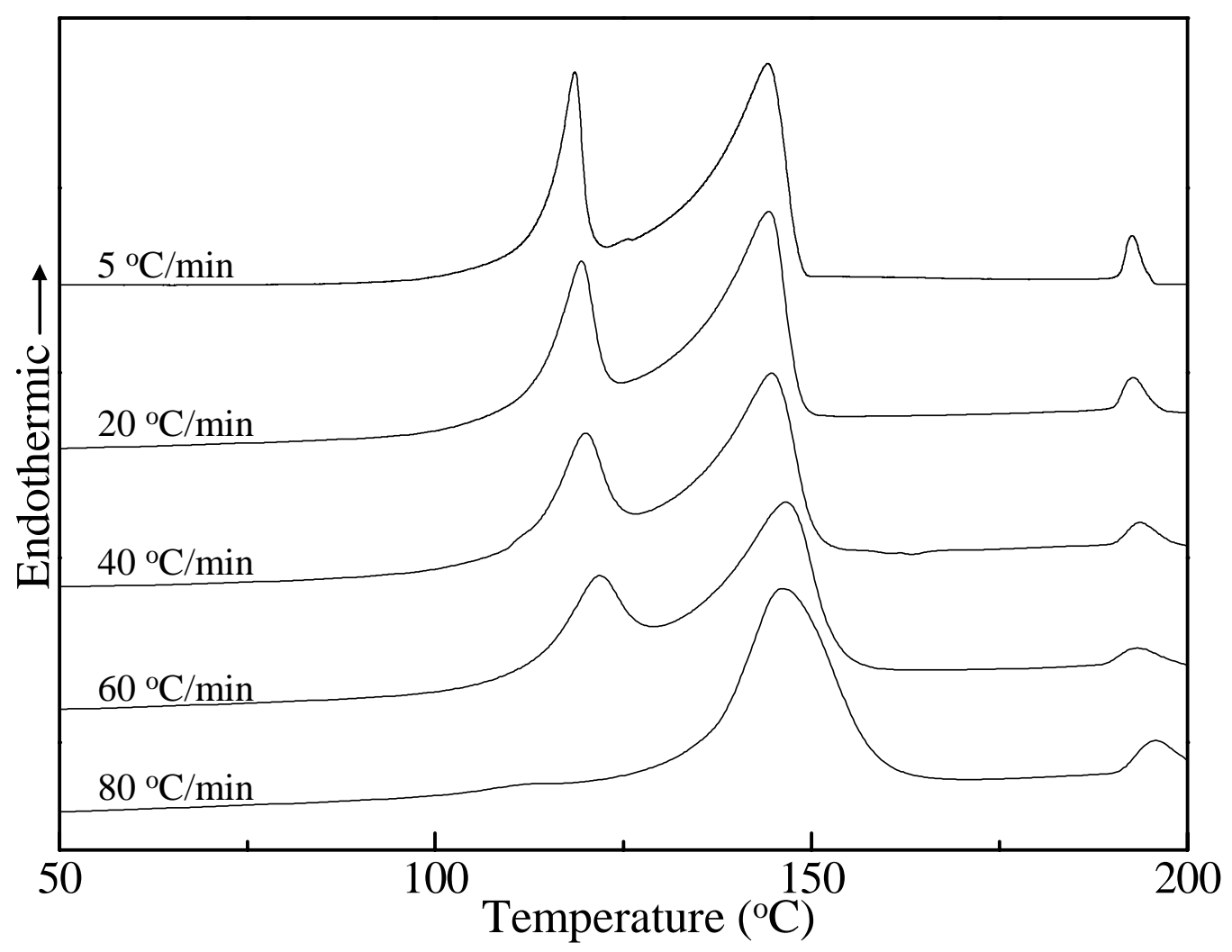

Figure S1. Thermograms of LC7 were performed in different heating rate. 


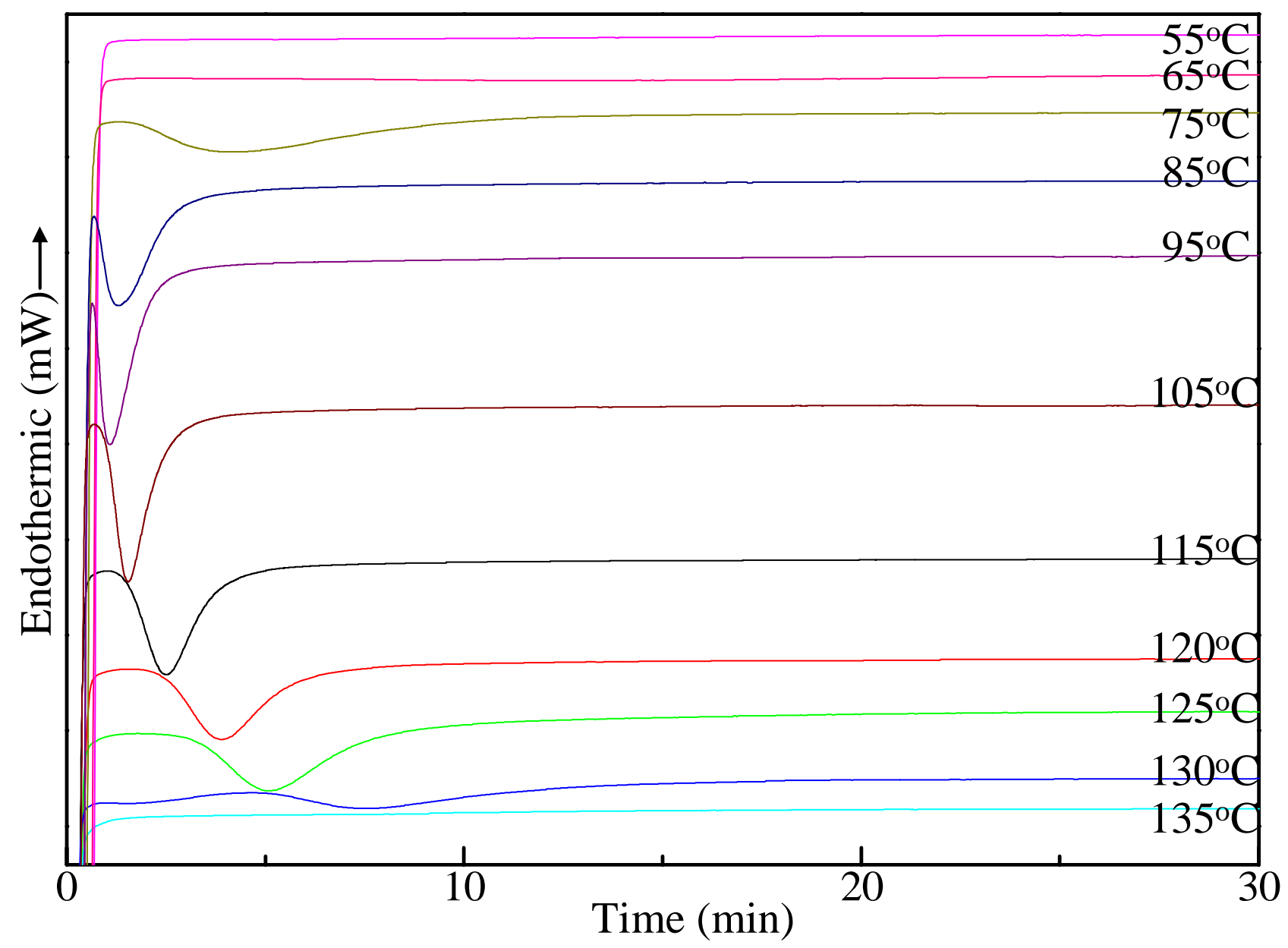

Figure S2. Thermogram of crystallization studies were performed in different temperature through 55 ${ }^{\circ} \mathrm{C}$ to $135{ }^{\circ} \mathrm{C}$ by LC11. A crystallization window was found between $65{ }^{\circ} \mathrm{C}$ and $130{ }^{\circ} \mathrm{C}$, and the maximum crystallization rate temperature was identified at $95^{\circ} \mathrm{C}$. 

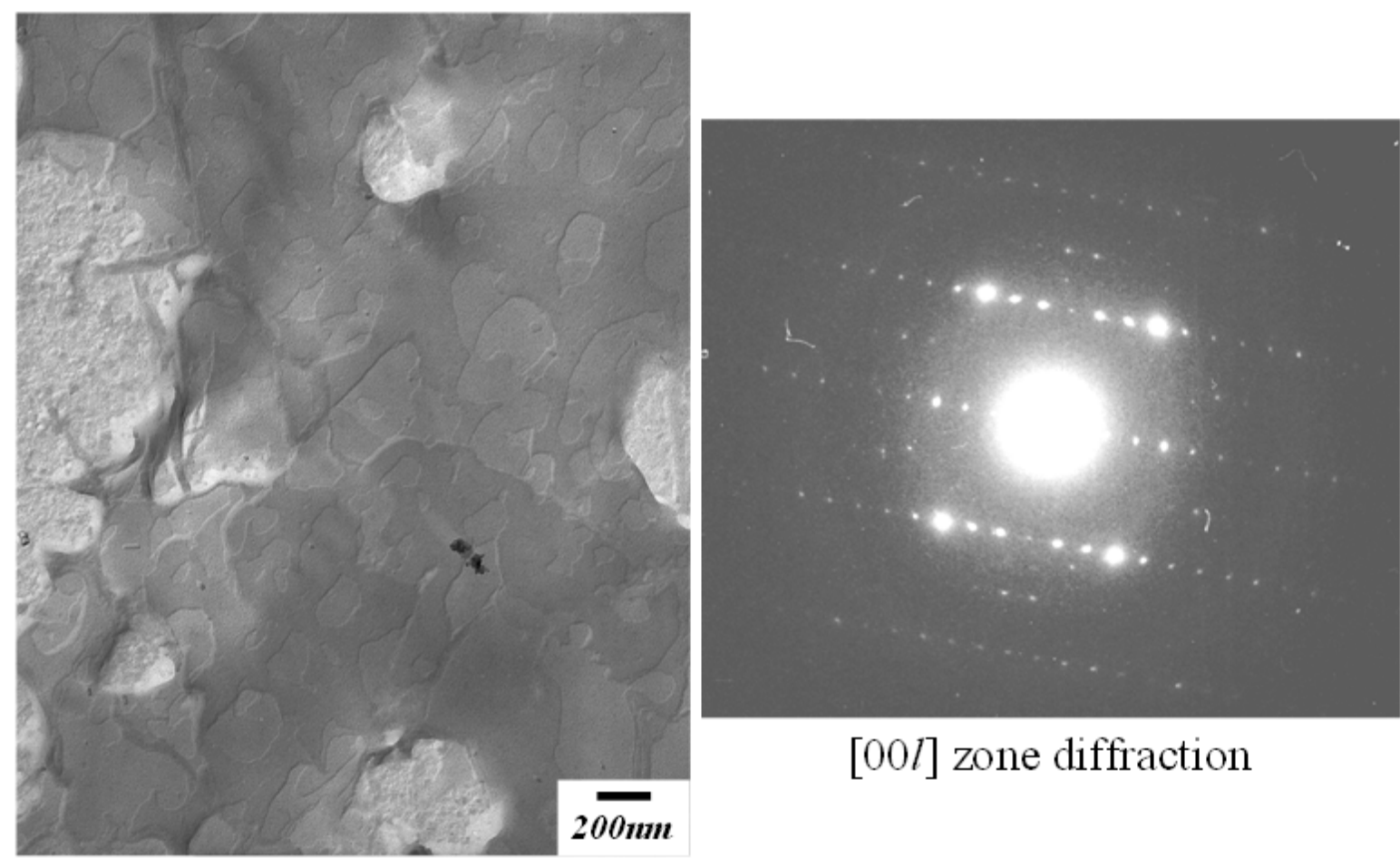

$[00 l]$ zone diffraction

Figure S3. Well-defined smectic-like structure observed by SAED [00l] zone diffraction. 

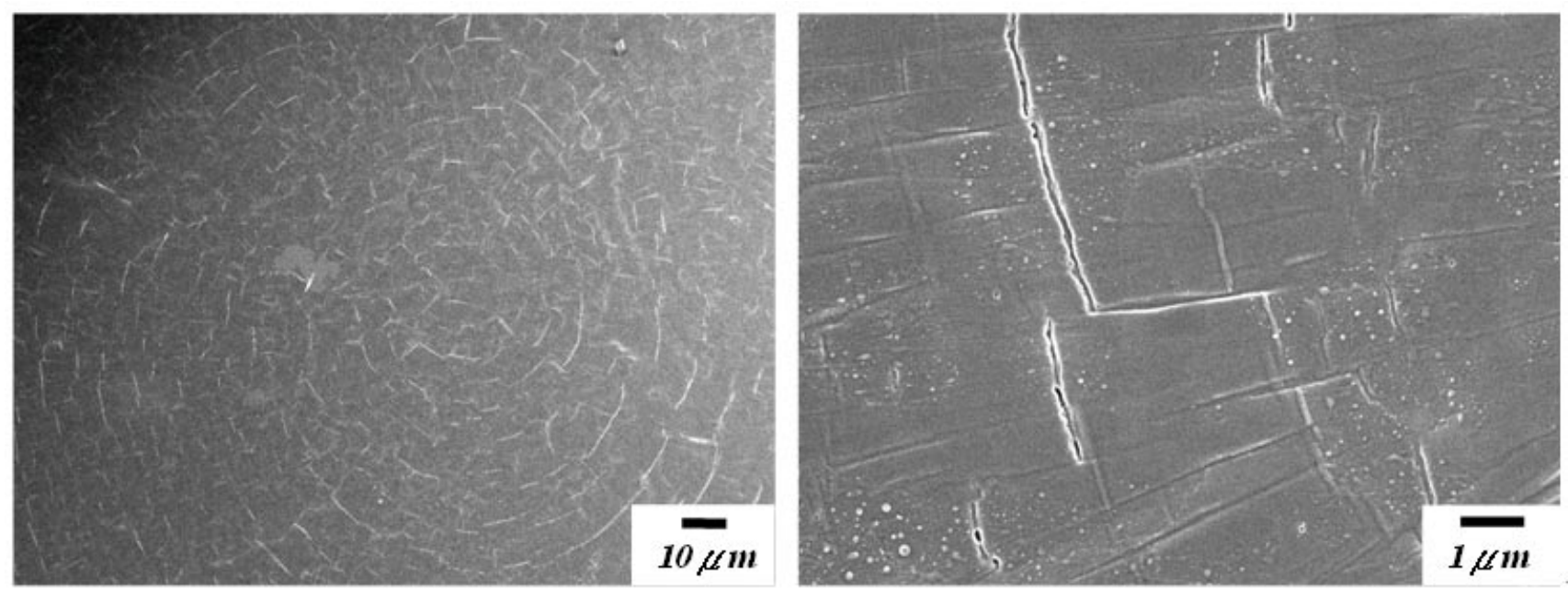

Figure S4. FESEM image of fractured banded texture at room temperature. 